\title{
A ZnTe THIN FILM MEMORY DEVICE
}

\author{
MARC BURGELMAN $\dagger$ \\ Laboratorium voor Elektronika en Meettechniek, Sint-Pietersnieuwstraat 41, B-9000 GENT, Belgium
}

(Received February 13, 1980)

\begin{abstract}
Thin ZnTe layers show memory switching characteristics. The electrical conduction in the non-ohmic off-state is shown to be due to Poole-Frenkel emission. An electrothermal model is proposed to explain the off-on transition; this model is confirmed by experiments. The characteristics of metal-ZnTe-metal sandwich structure as a memory device are investigated. The switching parameters are stable during a series of switching events, but the rather poor write-erase lifetime ( 200 cycles) still constitutes a handicap for the operation as a device.
\end{abstract}

\section{INTRODUCTION}

Memory switching phenomena were observed in many materials, both in bulk materials as in thin films, in amorphous and polycrystalline materials as well as in single crystals. ${ }^{1-3}$ Among all of these, the amorphous chalcogenide semiconductors are the most successful memory materials. There is a vast literature dealing with their chemical, electrical and optical properties and applications; for a review, see e.g. Tauc ${ }^{1}$ and Fritzsche. ${ }^{2}$ The use of these devices in memory arrays, however, necessitates a diode to be placed in series with each device, in order to avoid the sneak conducting path that occurs around a high-resistance device when three of its neighbours are in the low-resistance state. ${ }^{4}$ Hence the full silicon technology is needed, enlarged by the preparation and sputtering technology of the chalcogenide alloy. The chalcogenide memory devices are thus coming in direct competition with well established silicon RMM memory devices as MNOS and FAMOS. Perhaps because of the complicated technology, only one company succeeded in fabricating high-performance chalcogenide memory devices (i.e. Energy Conversion Devices Inc., Michigan (ECD)).

Memory switching in other materials, like polycrystalline II-VI films, were investigated only sporadically. The literature about switching in $\mathrm{ZnTe}$ thin films is restricted to one article by Ota and Takahashi in $1973 .{ }^{5}$ Our investigations are the first confirmation of the phenomena reported by Ota

$\lceil$ The author is indebted to the National Science Foundation (N.F.W.O) for a research assistantship. and Takahashi, and the first attempt to assess the applicability of $\mathrm{ZnTe}$ as a memory device and to gain insight into the physical mechanisms underlaying the switching behaviour in $\mathrm{ZnTe}$. $^{3,6}$

Our devices are made on a glass substrate by successive thermal evaporation of an $\mathrm{Al}$, a $\mathrm{ZnTe}$ and an $\mathrm{Al}$ layer; molybdenum contact masks were used to obtain the device geometry of Figure 1a. We intend to investigate which performances can be reached with the simple technology of an all thin film device, without having a recourse to the much more complicated silicon technology.

All our devices were initially in the non-ohmic, high resistive off-state (Figure 1b, branch ODA). The device switches to the ohmic, low-resistive on-state (branch $\mathrm{OBC}$ ) when a voltage exceeding the threshold voltage $V_{t h}$ is applied. The device switches back to the off-state when a current higher than the threshold current $I_{\text {th }}$ passes through the device. Let us now study these features in some more detail.

\section{ELECTRICAL CONDUCTION IN THE OFF-STATE}

Several high-field conduction mechanisms can give rise to a non-ohmic $\mathrm{I}-\mathrm{V}$ characteristic as observed in the off-state of ZnTe. It was stressed in the literature ${ }^{3,6,7}$ that the form of an I-V curve alone is a very misleading and insufficient criterion to judge the current transport. To investigate the conduction in our $\mathrm{ZnTe}$ layers, we represented our measurements in a $\log I$ and $\log (\mathrm{I} / \mathrm{V})$ vs. $\mathrm{V}, \sqrt{ } \mathrm{V}$ and $\log \mathrm{V}$ diagram; we then studied the temperature and thickness dependence of the slopes and intercepts 

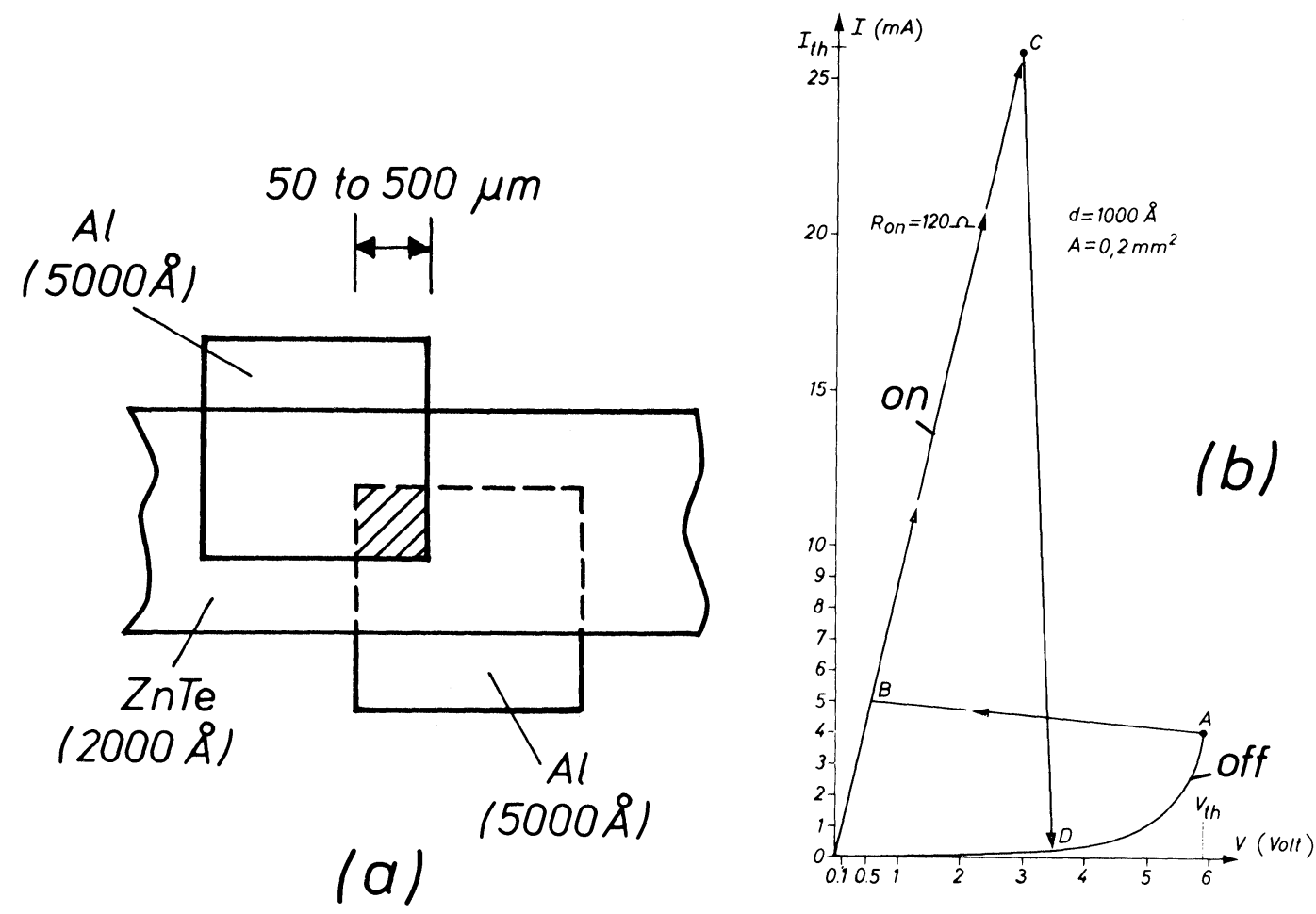

FIGURE 1 (a) Typical structure and (b) I-V characteristics with off- and on-state of a ZnTe memory device.

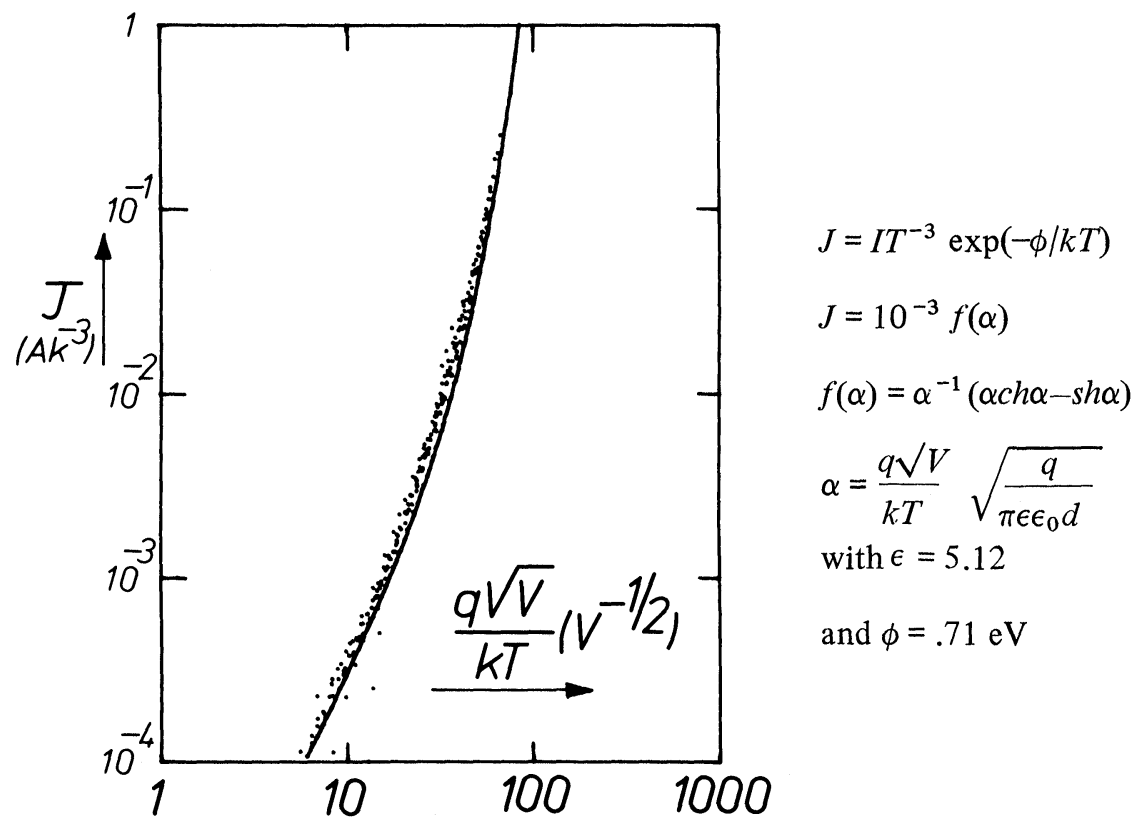

FIGURE 2 Hill-plot of the I-V measurements of a $\mathrm{ZnTe}$ layer at different ambient temperatures, showing Poole-Frenkel conduction. 
of the linear parts (if any) in these various diagrams, and compared the results with the theoretical predictions of several conduction mechanisms. ${ }^{3,6}$ In this way we were able to show that the conduction in our $\mathrm{ZnTe}$ layers is dominated by spherical uniform Poole-Frenkel emission of holes from acceptors lying some $0.7 \mathrm{eV}$ above the valence band edge. This is illustrated in Figure 2 where $242(\mathrm{I}, \mathrm{V})$ points measured at 11 different ambient temperatures between $-21^{\circ} \mathrm{C}$ and $86^{\circ} \mathrm{C}$ are represented in a normalized Hill-plot. ${ }^{8,9}$ All points are situated on one curve that was theoretically predicted by Hill $^{8}$ for the conduction mechanism under consideration. This is strong evidence that this mechanism dominates the conduction in our thin $\mathrm{ZnTe}$ films. Such a detailed investigation of current transport in thin $\mathrm{ZnTe}$ films was up to now absent in the literature.

\section{THE OFF-ON TRANSITION}

The strong dependence of the off-conductivity on the ambient temperature and the applied field gives rise at higher power levels to a Joule self-heating I-V characteristic with a region of negative differential resistance (NDR). Switching occurs when the NDR curve becomes tangential to the load line (Figure 3). We developed an electrothermal model to calculate the self-heating characteristics, using the conduction parameters measured at low currents and considering cooling by conduction, convection and radiation of the $\mathrm{ZnTe}$ on glass structure of Figure 1a. That model differs considerably from the models described in the literature for chalcogenide switches. There is a good agreement between the numerically calculated and

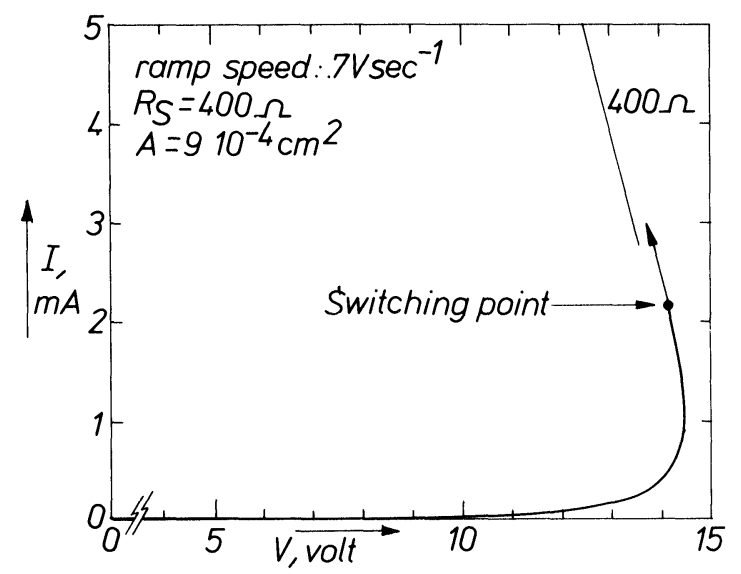

FIGURE 3 Measured off-characteristic with self-heating, showing turnover and NDR; the switching occurs in the direction of the load line. the measured characteristics concerning their general form and NDR behaviour, and the magnitude and the temperature and geometry dependence of the switching voltage. ${ }^{3}$

\section{THE ON-STATE AND THE ON-OFF TRANSITION}

After the off-on transition, the current is contracted in a hot filament, where the temperature rise, as calculated by our thermal model, is some hundreds of Kelvin above ambient. This high temperature allows the on-state to be locked within some milliseconds. The locked on-state is purely ohmic; its resistance $R_{o n}$ is virtually independent of the device geometry, but it is determined by the current carried in the lock-on period, and thus by the applied voltage and the series resistance during the off-on switch. $R_{\text {on }}$ shows a metallic behaviour with a temperature coefficient of $2.110^{-3} \mathrm{~K}^{-1}$.

There is still uncertainty about the nature of the locked on-state. X-ray analysis shows that our films have a crystallite size of some hundreds of ångström, so that an amorphous-crystalline transition, as is the case with the chalcogenides, is not appropriate here. A possible mechanism is phase separation giving rise to a $\mathrm{Zn}$ rich or to a Te rich on-filament. An X-ray emission analysis with a $2 \mu \mathrm{m}$ scanning electron beam however did not demonstrate a peak or dip of the $\mathrm{Zn}$ or $\mathrm{Te}$ concentration when scanning the device's area. Whether phase separation is not the lock-on mechanism, or the filament is less than $2 \mu \mathrm{m}$ can thus not be decided from the experiment.

The on-off switch occurs by applying a short current pulse higher than the threshold current to the device. The thermal model described by Burgelman $^{3}$ shows that a temperature rise of $1300 \mathrm{~K}$ within a few microseconds is possible in a small $(<1 \mu \mathrm{m})$ filament. This heating causes the on-filament whatever its nature is, to melt, so that the off-state can be restored by the subsequent quenching of the material.

\section{DEVICE CHARACTERISTICS}

Our devices show an off-on resistance ratio of $10^{4}$ to $10^{6}$. Neither speed requirements nor the device's lifetime are limitations in reading the device's memory state. The information can be maintained during at least two years without applied power. 


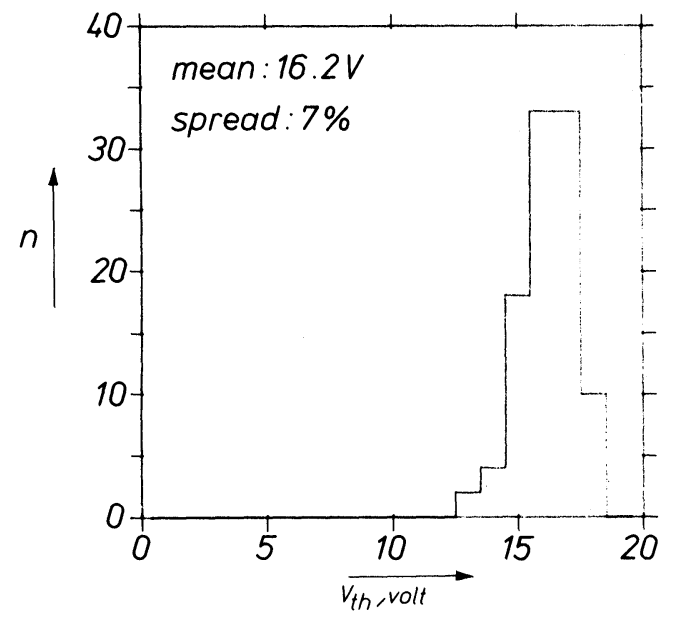

FIGURE 4 Histogram of the switching voltage $V_{\text {th }}$ for the first 100 switching cycles of an $\mathrm{Al}-\mathrm{ZnTe}-\mathrm{Al}$ device.

Writing information in a $\mathrm{ZnTe}$ device is slow: a few milliseconds are needed for the off-on switch. These considerations show that our devices behave as a read mostly memory (RMM). It was found ${ }^{3}$ that the write-erase lifetime could be increased by applying suitable switching pulses: a $15 \mathrm{~V}, 10 \mathrm{~ms}, 1 \mathrm{kohm}$ series resistance pulse is a good pulse for the off-on switch, whereas a $10 \mathrm{~V}, 10 \mu \mathrm{s}, 50 \mathrm{ohm}$ pulse is a good reset pulse. When switched under these conditions, the device's lifetime is some 200 switching cycles, the fluctuations of the switching parameters being less than $5 \%$ for $V_{t h}$ (Figure 4) and
$20 \%$ for $I_{t h}$. This performance is comparable to the characteristics of most of the chalcogenide memory devices fabricated by non-ECD workers, and is superior to the characteristics of $\mathrm{ZnTe}$ reported by Ota and Takahashi ${ }^{5}$ and to these of other II-VI materials.

\section{REFERENCES}

1. J. Tauc, (editor), Amorphous and Liquid Semiconductors (Plenum, New York 1974).

2. H. Fritzsche and S. Ovshinsky, "Amorphous semiconductors for switching, memory and imaging applications," IEEE Trans. Electron Devices, ED-20, 91-105 (1973).

3. Marc Burgelman, Onderzoek van elektrische-en schakeleigenschappen van dunne zinktelluride lagen, Doctorate's thesis, Ghent State University (1979) (in Dutch).

4. R. Neale and J. Aseltine, "The application of amorphous materials to computer memories", IEEE Trans. Electron Devices, ED-20, 195-205 (1973).

5. T. Ota and K. Takahashi, "Non-polarized memoryswitching characteristics of ZnTe thin films", SolidState Electron. 16, 1089-1096 (1973).

6. Marc Burgelman, "Conduction mechanisms in the offstate of thin ZnTe films", Solid-State Electron., 20, 523-528 (1977).

7. A. Owen and J. Robertson, "Electronic conduction and switching in amorphous semiconductors", IEEE Trans. Electron Devices, ED-20, 105-122 (1973).

8. R. M. Hill, "Poole-Frenkel conduction in amorphous solids", Phil. Mag., 23, 59-86 (1971).

9. H. Jonscher, and R. M. Hill, "Electrical conduction in disordered non-metallic films", Physics of Thin Films, 8, 169-249 (1975). 

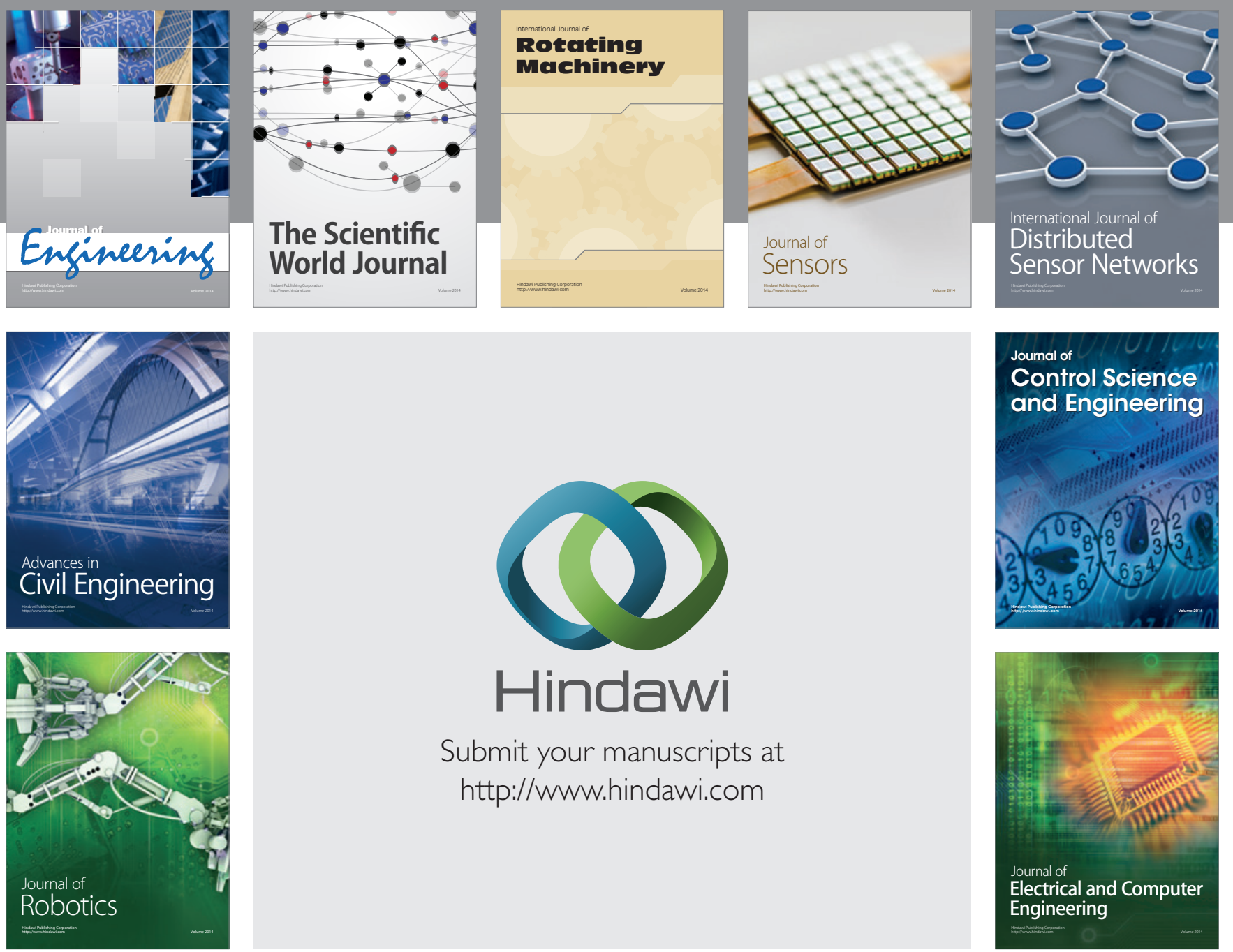

Submit your manuscripts at

http://www.hindawi.com
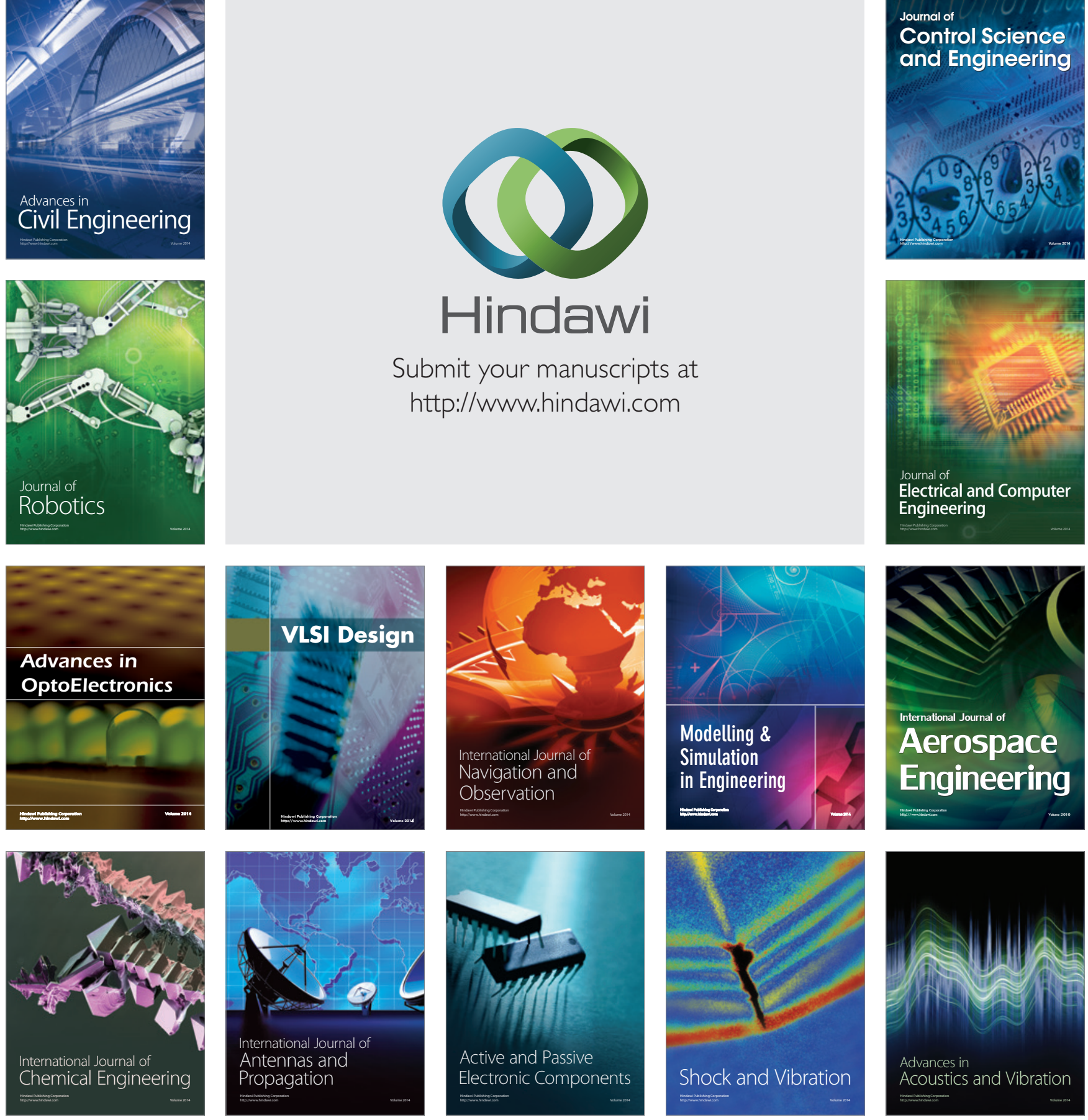